\title{
GUI Based Prediction of Diabetes Stages by Finding the Accuracy using Machine Learning Approach
}

\author{
${ }^{1}$ R.Shanthi B.Tech, ${ }^{2}$ V.Vinoth Kumar M.Tech, \\ ${ }^{I}$ Student M.E CSE Dept, ${ }^{2}$ Assistant professor Dept of Computer science and Engineering, \\ Gojan School of Business and Technology, Redhills, Chennai.
}

\begin{abstract}
In 2050, the world's diabetic patients will arrive at 642 million, which implies that one of the ten grownups later on is experiencing diabetes. Diabetes mellitus (DM) is characterized as a gathering of metabolic issues applying critical tension on human wellbeing around the world. DM is a persistent sickness portrayed by hyperglycemia and it might cause numerous inconveniences. To forestall this issue, to break down the given medical clinic dataset by directed AI technique(SMLT) with catch a few data resembles, variable ID, uni-variate examination, bi-variate and multi-variate investigation, missing worth therapies and dissect the information approval, information cleaning/getting ready and information perception will be done on the whole given dataset. Our analysis provides a comprehensive guide to sensitivity analysis of model parameters with regard to performance in prediction of diabetic patients by given attributes of dataset with evaluation of GUI based user interface diabetes attribute prediction. Additionally, it observes to lead an increase the highest accuracy in diabetic prediction of attributes by a significantly better classification report, identify the confusion matrix and to categorizing data from priority and the result shows that the effectiveness of the proposed machine learning algorithm technique can be compared with best accuracy with precision, Recall and F1 Score.
\end{abstract}

Keywords: Dataset, Machine learning Classification method, python, Prediction of Accuracy result.

\section{INTRODUCTION}

The scope of this project is to investigate a dataset of healthcare records for hospital sector using machine learning technique. We try to reduce this risk factor behind predicting from diabetes attributes of Indian hospitals to safe human so as to save lots of efforts and assets and to predict whether assigning the patient is affected or not.Major challenge is how to extract the information from these data because the amount is very large so some data mining and machine learning techniques can be used. additionally the normal result and extent of this undertaking is that if illness can be anticipated than early treatment can be given to the patients which can diminish the danger of life and save life of patients and cost to get treatment of infections can be decreased up somewhat by early acknowledgment. For this problem, a machine learning approach will train for prediction of disease. The rapid adoption of electronic health records has created a wealth of new data about patients, which is a goldmine for improving the understanding of human health. The above method is used to predict diseases using patient treatment history and healthiness data.

\section{BACKGROUND}

Type 2 diabetes mellitus (T2DM), being the fourth major cause of mortality worldwide, is a major public health problem that presents a significant rising prevalence [2]. Worldwide there are 415 million people living with diabetes and around 193 million people have undiagnosed diabetes. T2DM represents over $90 \%$ of patients with diabetes and prompts microvascular and full scale vascular inconveniences that cause significant mental and actual misery to the two patients and guardians, bringing about a gigantic weight on the medical services framework [3]. The range of complications arising from diabetes, due to the damaging nature of glucose molecules on the microand macro-vascular system includes: cardiovascular disease, coronary heart disease, blindness, nephropathy, peripheral neural disease, amputations, depression and erectile dysfunction [4,5]. Despite increasing knowledge regarding risk factors for type 2 diabetes and evidence for successful prevention programs, the incidence and prevalence of the disease continues to rise globally. It is estimated that 300 million people will have diabetes mellitus by 2025 and it will reach approximately 439 million by 2030, with an estimated prevalence of $7.7 \%$ by 2030 . According to recent estimations it will comprise $6.8 \%$ of all the causes of death [6], placing it as the seventh mayor cause of death in 2030 [7], according to World Health Organization 
(WHO) projections. In a population-based study in Spain, a prevalence of $13.8 \%$ was observed on a population over the age of 18, about half of those diabetes cases were not diagnosed [8]. The predominance of analyzed diabetes in the Basque Country has been demonstrated to be lower than the present at public level in Spain [9].The registered prevalence in the Basque Country was $9.12 \%$ in 2011, with an increasing pattern since 2008 [10]. Patients with T2DM have a higher total mortality rate, as well as risk of micro vascular and macrovascular events [11]. These complications reduce the quality of life of patients with T2DM [12], and lead to an increase in healthcare costs $[13,14]$. study show that glycemic control is the most main feature to prevent the organ damage and other complications of T2DM. The decrease of blood glucose levels in patients with diabetes decreases the mortality and morbidity rates significantly $[8,11,15]$. Additionally, it has been well established the association between blood pressure and lipid control with disease duration and the development and progression of diabetes related complications [16]. A few T2DM related inconveniences have been concentrated through various old style AI, profound learning and information mining strategies [17]. Identification of the risks factors associated with these complications is of great value to the clinical management of individuals with diabetes. Due to the high level of disability and incremental costs of the disease, it is necessary to investigate the main factors involved in the genesis of complications.

\section{PROPOSED METHOD}

The objective of this project is to investigate a dataset of healthcare records for hospital sector using machine learning technique. We try to reduce this risk factor behind predicting from diabetes attributes of Indian hospitals to safe human so as to save lots of efforts and assets and to predict whether assigning the patient is affected or not. Exploratory Data Analysis of diabetes attribute Prediction. Multiple datasets from different sources would be combined to form a generalized dataset, and then different machine learning algorithms would be applied to extract patterns and to obtain results with maximum accuracy. In this section of the report will load in the data, check for cleanliness, and then trim and clean given dataset for analysis. Make sure that the document steps carefully and justify for cleaning decisions. The data set collected for predicting given data is split into Training set and Test set. Generally, 7:3 ratios are applied to split the Training set and Test set. The Data Model which was created using Random Forest, logistic, Decision tree algorithms, K-Nearest Neighbor (KNN) and Support vector classifier (SVC) are applied on the Training set and based on the test result accuracy, Test set prediction is done.

\subsection{DATA FLOW DIAGRAM}

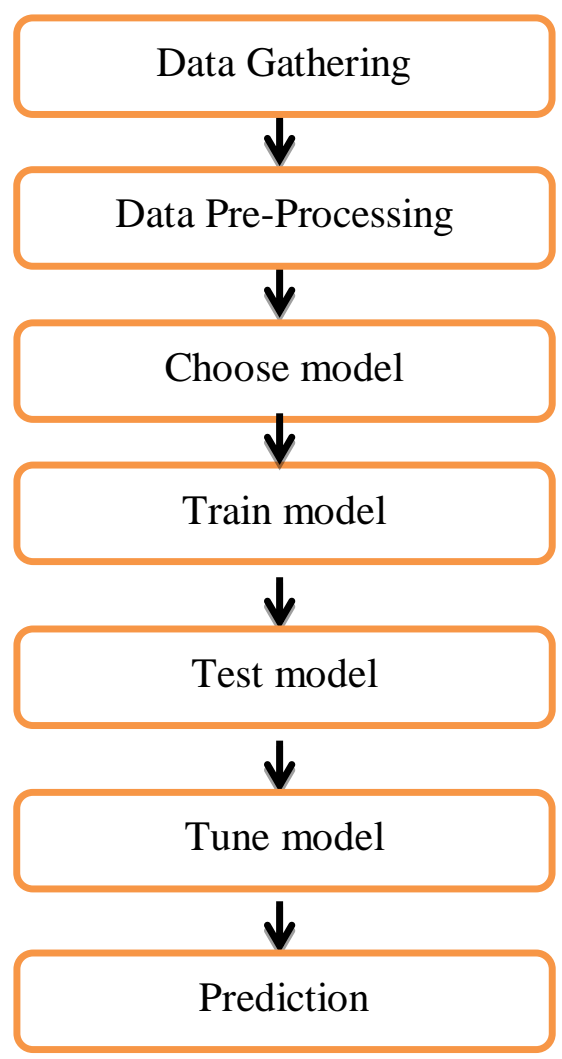




\section{MODULES DESCRIPTION}

\section{. Module-01:Validation technique:}

Validation techniques in machine learning are used to get the error rate of the Machine Learning (ML) model, which can be considered as close to the true error rate of the dataset. If the data volume is large enough to be representative of the population, you may not need the validation techniques. However, in real-world scenarios, to work with samples of data that may not be a true representative of the population of given dataset. To finding the missing value, duplicate value and description of data type whether it is float variable or integer. The sample of data used to provide an unbiased evaluation of a model fit on the training dataset while tuning model hyper parameters. The evaluation becomes more biased as skill on the validation dataset is incorporated into the model configuration. The validation set is used to evaluate a given model, but this is for frequent evaluation. It as machine learning engineers uses this data to fine-tune the model hyper parameters. Data collection, data analysis, and the process of addressing data content, quality, and structure can add up to a timeconsuming to-do list. During the process of data identification, it helps to understand your data and its properties; this knowledge will help you choose which algorithm to use to build your model. For example, time series data can be analyzed by regression algorithms; classification algorithms can be used to analyze discrete data. (For example to show the data type format of given dataset)

Data Validation/ Cleaning/Preparing Process:

\section{Module-02:Data visualization}

Data visualization is an important skill in applied statistics and machine learning. Statistics does indeed focus on quantitative descriptions and estimations of data. Data visualization provides an important suite of tools for gaining a qualitative understanding. This can be helpful when exploring and getting to know a dataset and can help with identifying patterns, corrupt data, outliers, and much more. With a little domain knowledge, data visualizations can be used to express and demonstrate key relationships in plots and charts that are more visceral and stakeholders than measures of association or significance. Data visualization and exploratory data analysis are whole fields themselves and it will recommend a deeper dive into some the books mentioned at the end. Sometimes data does not make sense until it can look at in a visual form, such as with charts and plots. Being able to quickly visualize of data samples and others is an important skill both in applied statistics and in applied machine learning. It will discover the many types of plots that you will need to know when visualizing data in Python and how to use them to better understand your own data.

\section{Module-03: \\ Logistic Regression:}

It is a statistical method for analysing a data set in which there are one or more independent variables that determine an outcome. The outcome is measured with a dichotomous variable (in which there are only two possible outcomes). The goal of logistic regression is to find the best fitting model to describe the relationship between the dichotomous characteristic of interest (dependent variable $=$ response or outcome variable) and a set of independent (predictor or explanatory) variables. Logistic regression is a Machine Learning classification algorithm that is used to predict the probability of a categorical dependent variable. In logistic regression, the dependent variable is a binary variable that contains data coded as 1 (yes, success, etc.) or 0 (no, failure, etc.). In other words, the logistic regression model predicts $\mathrm{P}(\mathrm{Y}=1)$ as a function of $\mathrm{X}$. Logistic regression Assumptions:

\section{Module-04: \\ Random Forest:}

Random forests or random decision forests are an ensemble learning method for classification, regression and other tasks, that operate by constructing a multitude of decision trees at training time and outputting the class that is the mode of the classes (classification) or mean prediction (regression) of the individual trees. Random decision forests correct for decision trees' habit of over fitting to their training set. Random forest is a type of supervised machine learning algorithm based on ensemble learning. Ensemble learning is a type of learning where you join different types of algorithms or same algorithm multiple times to form a more powerful prediction model. The random forest algorithm combines multiple algorithm of the same type i.e. multiple decision trees, resulting in a forest of trees, hence the name "Random Forest". The random forest algorithm can be used for both regression and classification tasks. 


\section{Module-05:K-nearest}

$K$-Nearest Neighbor is a supervised machine learning algorithm which stores all instances correspond to training data points in $\mathrm{n}$-dimensional space. When an unknown discrete data is received, it analyzes the closest $\mathrm{k}$ number of instances saved (nearest neighbors) and returns the most common class as the prediction and for realvalued data it returns the mean of $\mathrm{k}$ nearest neighbors. In the distance-weighted nearest neighbor algorithm, it weights the contribution of each of the $\mathrm{k}$ neighbors according to their distance using the following query giving greater weight to the closest neighbors.

\section{Module-06:Tkinter}

Tkinter is a python library for developing GUI (Graphical User Interfaces). We use the tkinter library for creating an application of UI (User Interface), to create windows and all other graphical user interface and Tkinter will come with Python as a standard package, it can be used for security purpose of each users.

\section{CONCLUSIONS}

The analytical process started from data cleaning and processing, missing value, exploratory analysis and finally model building and evaluation. Finding the patient stages and grade with parameter like accuracy, classification report and confusion matrix on public test set of given attributes by supervised machine learning algorithm method.

\subsection{FUTURE WORK}

Indian hospitals wants to automate the detecting the patient whether affecting diabetes or not by given attributes from eligibility process (real time).

To automate this process by show the prediction result in web application or desktop application in future. To optimize the work to implement in Artificial Intelligence environment.

\section{REFERENCES}

[1] Rammah Abohtyra, Yossi Chait, M. J. Germain, C.V. Hollot .". Individualization of Ultrafiltration in Hemodialysis “

[2] Xia Yu, Mudassir Rashid, Jianyuan Feng, . "Online Glucose Prediction Using Computationally Efficient Sparse Kernel Filtering Algorithms in Type-1 Diabetes “.

[3] Manu Goyal, . "Robust Methods for Real-time Diabetic Foot Ulcer Detection and Localization on Mobile Devices “

[4] Ali Al-Matouq1; Taous-meriem Laleg-Kirati2"Sparse Reconstruction of Glucose Fluxes using Continuous Glucose Monitors".

[5] Cecilia Saint-Pierre, MSc., Florencia Prieto"Team collaboration networks and multidisciplinary in diabetes care: Implications for patient outcomes

[6] Jonathan E. SHAW, Richard A. SICREE, and Paul Z. ZIMMET, "Global estimates of the prevalence of diabetes for 2010 and 2030 ," Diabetes research and clinical practice, vol. 87, pp. 4-14, 2010.

[7] Colin D. MATHERS and Dejan LONCAR, "Projections of global mortality and burden of disease from 2002 to 2030 ," PLoS medicine, vol. 3, p. 442,2006.

[8] Federico, SORIGUER, et al. "Prevalence of diabetes mellitus and impaired glucose regulation in Spain: the Diabetes Study," Diabetologia, vol. 55,pp. 88-93, 2012.

[9] J. M., ARTEAGOITIA, et al. "Incidence, prevalence and coronary heart disease risk level in known Type 2 diabetes: a sentinel practice network study in the Basque Country, Spain," Diabetologia, vol. 46, pp. 899-909,2003

[10] Edurne, ALONSO-MORÁN, et al. "Prevalence and quality of care indicators of type 2 diabetes in the population of the Basque Country (Spain)," Avances en Diabetología, vol. 31, pp. 72-79, 2015. 